(C) 2016 IEEE. Personal use of this material is permitted. Permission from IEEE must be obtained for all other uses, in any current or future media, including reprinting/republishing this material for advertising or promotional purposes, creating new collective works, for resale or redistribution to servers or lists, or reuse of any copyrighted component of this work in other works. 


\title{
Effective impedance modeling of metamaterial structures
}

\author{
Kokou B. Dossou ${ }^{1, *}$, Christopher G. Poulton ${ }^{1}$, and Lindsay C. Botten ${ }^{1,2}$ \\ ${ }^{1}$ Centre for Ultrahigh-Bandwidth Devices for Optical Systems (CUDOS), and School of Mathematical and Physical Sciences, University of Technology \\ Sydney, PO Box 123, Broadway, New South Wales 2007, Australia \\ ${ }^{2}$ National Computational Infrastructure, Australian National University, Canberra, Australia \\ *Corresponding author: Kokou.Dossou@uts.edu.au
}

Compiled December 16, 2015

\begin{abstract}
We present methods for retrieving the effective impedance of metamaterials from the Fresnel reflection coefficients at the interface between two semi-infinite media. The derivation involves the projection of rigorous modal expansions onto the dominant modes of the two semi-infinite media. It is shown that the effective impedance can also be written as a ratio of averaged field quantities. Thus a number of effective impedance formulas, previously obtained by field averaging techniques, can also be derived from the scattering-based formalism, by an appropriate choice of projection. Within the effective medium limit, it is observed that a simple semi-analytic modelling technique based on the effective impedance can be used to reliably compute the reflection coefficients of metamaterials over a wide range of incidence angles. We use this technique to model planar metamaterial waveguides or surface modes. ๑ 2015 Optical Society of America

OCIS codes: (260.2065) Effective medium theory; (160.3918) Metamaterials; (160.5298) Photonic crystals; (290.0290) Scattering.

http://dx.doi.org/10.1364/JOSAA.XX.XXXXXX
\end{abstract}

\section{INTRODUCTION}

Effective medium techniques, when applicable, can provide deep physical insight into the properties of metamaterials and lead to simplified analytical treatments of these heterogeneous structures. The study of the effective material parameters of metamaterials has attracted the interest of many researchers [16]. However, for a rigorous numerical simulation of metamaterials, general-purpose methods such as the finite-difference time-domain and the finite element methods are the preferred tools, although these methods become computationally intensive with the numerical simulation of three-dimensional metamaterials, especially when the metamaterials include a defect, e.g., metamaterial waveguides.

The solution of many important problems involves solving scattering problems between semi-infinite media. Field scattering at the interface between two semi-infinite media can be modelled elegantly by using the concept of effective impedance. The impedance can also provide insight into the existence of waveguide modes or surface modes in complex structures; for example the conditions for the existence of surface modes at the interface between two media rely on a change of sign of the effective dielectric permittivity or the effective magnetic permeability across the interface, and these effective material pa- rameters can be computed when the effective impedance and effective index are known. Once the effective impedance is known, the Fresnel reflection coefficients between two semiinfinite media can be determined using a simple impedance formula. Reciprocally, the effective impedance can also be retrieved once the Fresnel reflection coefficient is known, and many researchers have adopted such an approach for finding the effective impedance because of the availability of efficient semi-analytical methods or numerical methods for the determination of the Fresnel reflection and transmission coefficients at the interface between two semi-infinite media. The validity of the effective impedance modeling is usually restricted to regimes where the wave propagation in each medium is dominated by a single mode, and this usually requires low frequency operations (e.g., below the first Wood anomaly); otherwise, when several modes play a significant role in the wave propagation, the concept of generalized matrix impedance [5, 7] can be applied.

Here we propose an effective medium technique for metamaterials which exploits the strength of analytic techniques in modelling problems over an extended domain (e.g., scattering between semi-infinite media, waveguide modes, surface modes) and the efficiency of current numerical methods in com- 
puting the Bloch modes of bulk metamaterials (since the computational domain is reduced to a single unit cell). For the determination of the Fresnel reflection and transmission coefficients at the interface between two semi-infinite media, the fields in each medium are represented by Bloch mode expansions [5]. A biorthogonality property [5] between primal and adjoint modes, which is valid for lossless or lossy metamaterials, can be used to transform the field matching conditions into a system of linear equations. When the wave propagation in each medium is dominated by a single mode, the projection of the rigorous modal expansions onto the set of dominant modes leads to a truncated system of two linear equations with two unknowns, which can be solved analytically to obtain the approximate reflection and transmission coefficients. Then the relative effective impedance can be obtained from the reflection coefficient $R$ as $Z^{(2)} / Z^{(1)}=(1+R) /(1-R)$, and the mathematical expression for the relative effective impedance depends on the choice of the projection used in the truncation operations, although, in many cases, the actual value of the impedance tends to be only weakly dependent on projection type.

The existence of a number of different effective impedance formulas also reflects the fact that there are many variations in the implementation of the mode-matching techniques. The mode-matching equations are obtained by projecting the modal expansions on either side of an interface onto a set of test functions and several choices of test functions have been proposed and validated. The set of test functions can consist of both the electric fields and magnetic fields of the modes from only one side of the interface [8], and the mathematical expressions for the coefficients of the mode-matching equations depend on which side is chosen. It is also possible to choose the electric fields of the modes from one side of the interface and the magnetic fields of the modes from the other side [9], again the mathematical presentation depends on the type of electromagnetic field each side gets.

There is another class of homogenization methods, known as field-averaging methods [10], where the effective impedance can be retrieved as a ratio of averaged field quantities. It was shown [11] that a definition of effective impedance based on eigen-field averaging [12] was equivalent to another one which was based on the Fresnel reflection coefficient. Such equivalence properties can be useful, for instance, the mathematical and physical justifications of one type of method can be extended to the other and vice versa. We will show that two other field-averaged impedance definitions $[13,14]$ can also be obtained from a Fresnel-based impedance method by projecting the mode-matching equations in an appropriate way.

The paper is organized as follows. The effective impedance is defined in the next section; we will see that it can be written as a ratio between averaged field quantity and the precise form of the effective impedance also depends on the chosen generalized inner product. In Section 3, the concept of effective impedance will be applied to the modelling of metamaterials. In Section 4, as a validation test, we will model an array of cylinder holes perforated on a perfectly conducting material and investigate the existence of surface mode at the interface between the metamaterials and vacuum. Finally, an example of lossy metamaterials will be analyzed in Section 5 and the dispersion curves of a metamaterial waveguide will be computed using the effective medium technique and a comparison will be made against the dispersion curves obtained with a finite element method.

\section{THE EFFECTIVE IMPEDANCE}

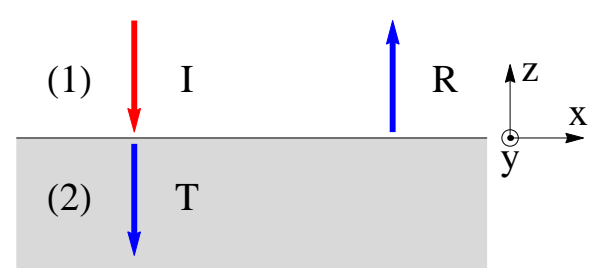

Fig. 1. Illustration of an incidence from a semi-infinite medium (1) into a semi-infinite medium (2). The symbols $I, R$ and $T$ represent respectively the incident, reflected and transmitted fields.

We consider the problem of wave incidence from a periodic semi-infinite medium (1) into a periodic semi-infinite medium (2) (see Fig. 1). The mathematical treatment presented in this section is very general with respect to the material geometry, and can be applied, for instance, when both media (1) and (2) are metamaterials. It is assumed that a single mode plays a dominant role in the field propagation in each medium; such an assumption is typically valid for the regimes where the wavelength is much longer than the characteristic length (e.g., lattice constant) of media (1) and (2). The dominant Bloch modes of the media (1) and (2) are respectively denoted $\left(\boldsymbol{E}^{(1)}, \boldsymbol{H}^{(1)}\right)$ and $\left(\boldsymbol{E}^{(2)}, \boldsymbol{H}^{(2)}\right)$ and, when required, the plus and minus signs in superscript indicate the direction of propagation of a mode.

We assume that the downward propagating mode $\left(\boldsymbol{E}^{(1)-}, \boldsymbol{H}^{(1)-}\right)$ is incident over the medium (2) and the reflected field is dominated by the contribution from the upward propagating mode $\left(\boldsymbol{E}^{(1)+}, \boldsymbol{H}^{(1)+}\right)$ while the transmitted field is dominated by the mode $\left(\boldsymbol{E}^{(2)-}, \boldsymbol{H}^{(2)-}\right)$. If $R$ and $T$ are respectively the reflection and transmission coefficients, the tangential field continuity at the interface between the semi-infinite media can be written as

$$
\begin{gathered}
\boldsymbol{E}_{\perp}^{(1)-}+R \boldsymbol{E}_{\perp}^{(1)+}+\Delta \mathcal{E}_{\perp}^{(1)}=T \boldsymbol{E}_{\perp}^{(2)-}+\Delta \mathcal{E}_{\perp}^{(2)}, \\
\boldsymbol{H}_{\perp}^{(1)-}+R \boldsymbol{H}_{\perp}^{(1)+}+\Delta \mathcal{H}_{\perp}^{(1)}=T \boldsymbol{H}_{\perp}^{(2)-}+\Delta \mathcal{H}_{\perp}^{(2)},
\end{gathered}
$$

where $\left(\Delta \mathcal{E}^{(1)}, \Delta \mathcal{H}^{(1)}\right)$ and $\left(\Delta \mathcal{E}^{(2)}, \Delta \mathcal{H}^{(2)}\right)$ are the contribution, of the Bloch modes different from $\left(\boldsymbol{E}^{(1)}, \boldsymbol{H}^{(1)}\right)$ and $\left(\boldsymbol{E}^{(2)}, \boldsymbol{H}^{(2)}\right)$, to the electromagnetic field on either side of the interface.

The presentation can be conveniently simplified by assuming that the unit cell is up-down symmetric, i.e., its geometry and material distributions are invariant under the transformation $(x, y, z) \mapsto(x, y,-z)$. Under this assumption, the two counter-propagating modes $\left(\boldsymbol{E}^{(n)+}, \boldsymbol{H}^{(n)+}\right)$ and $\left(\boldsymbol{E}^{(n)-}, \boldsymbol{H}^{(n)-}\right)$ can be chosen such that [5]

$$
\begin{aligned}
\boldsymbol{E}^{(n)-} & =\left(\boldsymbol{E}_{\perp}^{(n)-}, \boldsymbol{E}_{z}^{(n)-}\right)=\left(\boldsymbol{E}_{\perp}^{(n)+},-\boldsymbol{E}_{z}^{(n)+}\right), \\
\boldsymbol{H}^{(n)-} & =\left(\boldsymbol{H}_{\perp}^{(n)-}, \boldsymbol{H}_{z}^{(n)-}\right)=\left(-\boldsymbol{H}_{\perp}^{(n)+}, \boldsymbol{H}_{z}^{(n)+}\right) .
\end{aligned}
$$

The tangential field continuity conditions Eqs. (1)-(2) become

$$
\begin{aligned}
(1+R) \boldsymbol{E}_{\perp}^{(1)}+\Delta \mathcal{E}_{\perp}^{(1)} & =T \boldsymbol{E}_{\perp}^{(2)}+\Delta \mathcal{E}_{\perp}^{(2)} \\
(1-R) \boldsymbol{H}_{\perp}^{(1)}+\Delta \mathcal{H}_{\perp}^{(1)} & =T \boldsymbol{H}_{\perp}^{(2)}+\Delta \mathcal{H}_{\perp}^{(2)} .
\end{aligned}
$$




\section{A. The adjoint modes and biorthogonality relations}

Orthogonality relations are very useful in determining the coefficients of a modal expansion in terms of the modes of an orthogonal basis. With Hermitian problems, the orthogonality properties involve a set of modes and their complex conjugate. For the cases of non-Hermitian problems, the modal expansion can be handled efficiently by using adjoint modes and a detailed derivation of the biorthogonality relations between primal and adjoint modes can be found in Appendix A of [6].

Let $\left(\boldsymbol{E}_{n}, \boldsymbol{H}_{n}\right)$, for $n=1,2,3, \ldots$, be the Bloch modes (primal modes) of a metamaterial structure. The primal mode $\left(\boldsymbol{E}_{n}, \boldsymbol{H}_{n}\right)$ is supposed to have a fixed transverse wavevector $k_{\perp}=\left(k_{x}, k_{y}\right)$ and a fixed time dependence $e^{-i \omega t}$; for example, with the problem of incidence by a plane wave, $\left(k_{x}, k_{y}\right)$ is the transverse wave vector of the incident plane wave and $\omega$ is the angular frequency of the incident wave. The notation $\left(\boldsymbol{E}_{n}^{\dagger}, \boldsymbol{H}_{n}^{\dagger}\right)$ will denote the adjoint mode of a Bloch mode $\left(\boldsymbol{E}_{n}, \boldsymbol{H}_{n}\right)$. The adjoint Bloch mode $\left(\boldsymbol{E}_{n}^{\dagger}, \boldsymbol{H}_{n}^{\dagger}\right)$ is defined as a mode which satisfies the same wave equation as the primal Bloch mode $\left(\boldsymbol{E}_{n}, \boldsymbol{H}_{n}\right)$ but has the opposite transverse wavevector component $\boldsymbol{k}_{\perp}^{\dagger}=-k_{\perp}=$ $-\left(k_{x}, k_{y}\right)$ and the opposite time dependence, i.e., $e^{i \omega t}$. The set of adjoint modes and primal modes form a biorthogonal system $[5,6]$ :

$$
\begin{aligned}
\int_{\Omega} \boldsymbol{E}_{n} \cdot\left(\boldsymbol{e}_{z} \times \boldsymbol{H}_{m}^{\dagger}\right) d A & =\delta_{n m}, \\
\int_{\Omega} \boldsymbol{E}_{n}^{\dagger} \cdot\left(\boldsymbol{e}_{z} \times \boldsymbol{H}_{m}\right) d A & =-\delta_{n m},
\end{aligned}
$$

where the integration domain $\Omega$ is a transverse interface of a unit cell, e.g., the top interface.

Note that the biorthogonality relations Eqs. (7) and (8) are valid under the assumption that each pair of counterpropagating Bloch modes $\left(\boldsymbol{E}^{(n)+}, \boldsymbol{H}^{(n)+}\right)$ and $\left(\boldsymbol{E}^{(n)-}, \boldsymbol{H}^{(n)-}\right)$ can be normalized to take the form given by Eqs (3) and (4), which normally requires the use of up-down symmetric unit cells. The cases where the unit cell does not have the required symmetry can be handled by introducing the generalized inner product [15]

$$
\left\langle\left(\boldsymbol{E}_{n}^{\dagger}, \boldsymbol{H}_{n}^{\dagger}\right) \mid\left(\boldsymbol{E}_{m}, \boldsymbol{H}_{m}\right)\right\rangle=\int_{\Omega}\left(\boldsymbol{E}_{m} \times \boldsymbol{H}_{n}^{\dagger}-\boldsymbol{E}_{n}^{\dagger} \times \boldsymbol{H}_{m}\right) \cdot \boldsymbol{e}_{z} d A .
$$

We note in passing that although a general orthogonality framework was used in [15], further symmetry assumptions were needed in order to define the Bloch impedance of the metamaterial, i.e., the metamaterial is "mirror-symmetric with respect to both $x$ and $y$ direction" and the unit cell is "mirror symmetric with respect to $z$ direction" (see Eqs. (19) and (20) in [15]).

In general the adjoint modes can be obtained by solving the adjoint eigenproblem. However for a normal incidence, the direct calculation of the adjoint modes can be avoided. Indeed, since the adjoint and primal modes have the same transverse wavevector $\boldsymbol{k}_{\perp}^{\dagger}=\boldsymbol{k}_{\perp}=(0,0)$ but opposite time dependence, it follows that the adjoint mode can be taken as

$$
\left(\boldsymbol{E}_{n}^{\dagger}, \boldsymbol{H}_{n}^{\dagger}\right)=\left(\boldsymbol{E}_{n},-\boldsymbol{H}_{n}\right) \text {. }
$$

Symmetry consideration can also be used to obtain the adjoint modes. For instance when the periodic structure is invariant under the transformation $(x, y, z) \mapsto(-x,-y, z)$, the adjoint modes can be derived directly from the primal modes as [5]:

$$
\begin{aligned}
\boldsymbol{E}_{n}^{\dagger}(x, y, z) & =\left(\boldsymbol{E}_{n, \perp}(-x,-y, z),-E_{n, z}(-x,-y, z)\right), \\
\boldsymbol{H}_{n}^{\dagger}(x, y, z) & =\left(-\boldsymbol{H}_{n, \perp}(-x,-y, z), H_{n, z}(-x,-y, z)\right) .
\end{aligned}
$$

For the computation of the Bloch modes, we have used numerical methods $[9,16]$ which take either the electric field $E$ or the magnetic field $\boldsymbol{H}$ as the main unknown while the other electromagnetic field is obtained analytically from the Maxwell's equations. For instance, when the electric field $E$ is the primary unknown, the magnetic field $\boldsymbol{H}$ associated with the electric field $\boldsymbol{E}$ can be obtained from the Maxwell's equations as

$$
Z_{0} \boldsymbol{H}=\frac{-i}{k_{0} \mu} \nabla \times \boldsymbol{E} .
$$

In Eq. (13), $k_{0}$ is the free-space wavenumber, $\mu$ is the relative magnetic permeability, the symbol $Z_{0}$ is the impedance of free space $Z_{0}=\sqrt{\mu_{0} / \varepsilon_{0}}$, where $\varepsilon_{0}$ and $\mu_{0}$ denote respectively the absolute dielectric permittivity and magnetic permeability of the vacuum. For the remainder of the text, the magnetic field $\boldsymbol{H}$ will be rescaled as $Z_{0} \boldsymbol{H} \rightarrow \boldsymbol{H}$. Note that, with the rescaled magnetic field, the characteristic impedance [17] of the vacuum becomes $Z=E / H=1$.

\section{B. Derivation of the effective impedance}

We now take the scalar product of Eqs. (5) and (6), respectively, by $\boldsymbol{z} \times \boldsymbol{H}^{(1) \dagger}$ and $\boldsymbol{z} \times \boldsymbol{E}^{(2) \dagger}$, and apply the biorthogonality relations Eqs. (7) and (8); in particular we have

$$
\int_{\Omega} \Delta \mathcal{E}^{(1)} \cdot\left(\boldsymbol{e}_{z} \times \boldsymbol{H}^{(1) \dagger}\right) d A=\int_{\Omega} \Delta \mathcal{H}^{(2)} \cdot\left(\boldsymbol{z} \times \boldsymbol{E}^{(2) \dagger}\right) d A=0 .
$$

Under the condition that the scalar products are dominated by the contribution from the modes $\left(\boldsymbol{E}^{(1)}, \boldsymbol{H}^{(1)}\right)$ and $\left(\boldsymbol{E}^{(2)}, \boldsymbol{H}^{(2)}\right)$, we can also neglect the two remaining integrals

$$
\int_{\Omega} \Delta \mathcal{E}^{(2)} \cdot\left(\boldsymbol{e}_{z} \times \boldsymbol{H}^{(1) \dagger}\right) d A \text { and } \int_{\Omega} \Delta \mathcal{H}^{(1)} \cdot\left(\boldsymbol{z} \times \boldsymbol{E}^{(2) \dagger}\right) d A,
$$

involving $\left(\Delta \mathcal{E}^{(1)}, \Delta \mathcal{H}^{(1)}\right)$ or $\left(\Delta \mathcal{E}^{(2)}, \Delta \mathcal{H}^{(2)}\right)$; we are then led to the following truncated system of linear equations:

$$
\begin{aligned}
1+R & =J^{\left(k_{x}, k_{y}\right)} T, \\
K^{\left(k_{x}, k_{y}\right)}(1-R) & =-T,
\end{aligned}
$$

where $J^{\left(k_{x}, k_{y}\right)}$ and $K^{\left(k_{x}, k_{y}\right)}$ are the overlap integrals

$$
\begin{aligned}
J^{\left(k_{x}, k_{y}\right)} & =\int_{\Omega} \boldsymbol{E}^{(2)} \cdot\left(\boldsymbol{z} \times \boldsymbol{H}^{(1) \dagger}\right) d A, \\
K^{\left(k_{x}, k_{y}\right)} & =\int_{\Omega} \boldsymbol{E}^{(2) \dagger} \cdot\left(\boldsymbol{z} \times \boldsymbol{H}^{(1)}\right) d A=-J^{\left(-k_{x},-k_{y}\right)},
\end{aligned}
$$

and $k_{0 \perp}=\left(k_{x}, k_{y}\right)$ is the transverse wave vector of the incident plane wave. The minus sign in the relation $K^{\left(k_{x}, k_{y}\right)}=$ $-J^{\left(-k_{x},-k_{y}\right)}$ comes from the fact that the primal modes and adjoint modes have opposite time dependence, $e^{-i \omega t}$ and $e^{i \omega t}$ respectively. By solving this linear system, we obtain the following approximated Fresnel reflection and transmission coefficients $R$ and $T$ :

$$
\begin{aligned}
R & =\frac{J^{\left(k_{x}, k_{y}\right)} J^{\left(-k_{x},-k_{y}\right)}-1}{J^{\left(k_{x}, k_{y}\right)} J^{\left(-k_{x},-k_{y}\right)}+1}, \\
T & =\frac{2 J^{\left(-k_{x},-k_{y}\right)}}{J^{\left(k_{x}, k_{y}\right)} J^{\left(-k_{x},-k_{y}\right)}+1} .
\end{aligned}
$$

When both media (1) and (2) are uniform isotropic media, their plane wave basis functions have, up to a multiplicative factor, the same transverse components $\left(\boldsymbol{E}_{n, \perp}, \boldsymbol{H}_{n, \perp}\right)$; in particular 
the biorthogonality property implies that $\Delta \mathcal{E}^{(2)}$ is orthogonal to $\boldsymbol{e}_{z} \times \boldsymbol{H}^{(1) \dagger}$ and $\Delta \mathcal{H}^{(1)}$ is orthogonal to $\boldsymbol{z} \times \boldsymbol{E}^{(2) \dagger}$ so that the values of the two integrals in Eq. (15) are zeros. In such cases, the relations Eqs. (16) and (17) are exact relations which do not involve any approximation and the formulas Eqs. (20) and (21) for the reflection and transmission coefficients are also exact formulas. Also for cases where the transverse component of the modes of medium (2) can be seen as a small perturbation to the transverse component of the modes of the medium (1), the formulas Eqs. (20) and (21) can be accurate over a relatively large range of frequencies. form

The Fresnel reflection coefficient Eq. (20) has the general

$$
R=\frac{Z^{(2)}-Z^{(1)}}{Z^{(2)}+Z^{(1)}}=\frac{Z^{(2)} / Z^{(1)}-1}{Z^{(2)} / Z^{(1)}+1}
$$

accordingly, the coefficient

$$
Z_{\text {rel }}=Z_{\text {rel }}\left(k_{x}, k_{y}\right)=J^{\left(k_{x}, k_{y}\right)} J^{\left(-k_{x},-k_{y}\right)}
$$

represents the relative effective impedance $Z^{(2)} / Z^{(1)}$. The relative effective impedance $Z_{\text {rel }}$ depends on the transverse wavevector $\left(k_{x}, k_{y}\right)$ and, if necessary, it can be written explicitly as a function of $\left(k_{x}, k_{y}\right)$, i.e., $Z_{\text {rel }}\left(k_{x}, k_{y}\right)$.

\section{Effective impedance as field averaging}

We have defined in Eq. (23) the relative effective impedance $Z_{\text {rel }}=Z^{(2)} / Z^{(1)}$ between two media, here we want to define the absolute effective impedance $Z^{(2)}$ of the medium (2), assuming that the incident medium (1) is, for instance, vacuum. The mode $\left(\boldsymbol{E}^{(1)}, \boldsymbol{H}^{(1)}\right)$ of the medium (1) has a simple analytical expression and the substitution into the overlap integrals of Eq. (23) leads to an integral representation of $Z_{\text {rel }}$ where only the mode of medium (2) appears explicitly. We shall see that this integral representation can be written as a ratio between two averaged field quantities, which means that our effective impedance formalism, which is originally based on a Fresnel scattering technique, can also be seen as a field averaging technique. The effective wave impedance $Z_{\text {eff }}^{(1)}$ of the medium (1) can also be obtained analytically as a ratio of the transverse field components: $Z_{\text {eff }}^{(1)}=E_{\perp}^{(1)} / H_{\perp}^{(1)}$ [17].

For an incidence from vacuum, for instance, by an $E_{y^{-}}$ polarized plane wave, the mode $\left(\boldsymbol{E}^{(1)}, \boldsymbol{H}^{(1)}\right)$ takes the form

$$
\begin{aligned}
\boldsymbol{E}^{(1)} & =\left(0, E_{y}^{(1)}, 0\right) e^{i\left(k_{x} x+k_{z}^{(1)} z\right)} \\
\boldsymbol{H}^{(1)} & =\left(H_{x}^{(1)}, 0, H_{z}^{(1)}\right) e^{i\left(k_{x} x+k_{z}^{(1)} z\right)} .
\end{aligned}
$$

The relative effective impedance $Z_{\text {rel }}=J^{\left(k_{x}, 0\right)} J^{\left(-k_{x}, 0\right)}$ becomes

$$
\begin{aligned}
Z_{\text {rel }} & =\left(H_{x}^{(1)}\right)^{2} \int_{\Omega} E_{y}^{(2)}(x, y, 0) e^{-i k_{x} x} d x d y \\
& \times \int_{\Omega} E_{y}^{(2)}(-x, y, 0) e^{i k_{x} x} d x d y,
\end{aligned}
$$

where Eq. (11) is used to express the adjoint mode component $E_{y}^{(2) \dagger}(x, y, 0)$ as $E_{y}^{(2)}(-x, y, 0)$; note that since $k_{y}=0$ it is not necessary to replace the variable $y$ by $-y$. The biorthogonality relation Eq. (7) shows that, for $j=1,2$, the overlap integral between $\boldsymbol{E}_{\perp}^{(j)}(x, y, 0)$ and $\boldsymbol{H}_{\perp}^{(j)}{ }^{\dagger}(x, y, 0)=-\boldsymbol{H}_{\perp}^{(j)}(-x, y, 0)$ is equal to one, it follows that Eq. (26) can be written as

$$
\begin{aligned}
Z_{\mathrm{rel}} & =\frac{\left(H_{x}^{(1)}\right)^{2}}{E_{y}^{(1)}\left(-H_{x}^{(1)}\right)|\Omega|} \\
& \times \frac{\int_{\Omega} E_{y}^{(2)}(x, y, 0) e^{-i k_{x} x} d x d y \int_{\Omega} E_{y}^{(2)}(-x, y, 0) e^{i k_{x} x} d x d y}{\int_{\Omega} E^{(2)}(x, y, 0) \cdot\left(\boldsymbol{e}_{z} \times\left(-H^{(2)}(-x, y, 0)\right)\right) d A},
\end{aligned}
$$

where $|\Omega|$ denotes the area of the interface $\Omega$. From Eq. (13) we have $H_{x}^{(1)}=-E_{y}^{(1)} k_{z}^{(1)} / k_{0}$ and the wave impedance of the medium (1) is then [17] $Z_{\text {eff }}^{(1)}=E_{y}^{(1)} /\left(-H_{x}^{(1)}\right)=k_{0} / k_{z}^{(1)}$. The effective wave impedance of the medium (2) is

$$
\begin{aligned}
Z_{\mathrm{eff}}^{(2)} & =Z_{\mathrm{rel}} Z_{\mathrm{eff}}^{(1)} \\
& =\frac{\left\langle E_{y}^{(2)}(x, y, 0) e^{-i k_{x} x}\right\rangle\left\langle E_{y}^{(2)}(-x, y, 0) e^{i k_{x} x}\right\rangle}{\left\langle\left(\boldsymbol{E}^{(2)}(x, y, 0) \times \boldsymbol{H}^{(2)}(-x, y, 0)\right) \cdot \boldsymbol{e}_{z}\right\rangle},
\end{aligned}
$$

where the operator $\langle F\rangle$ denotes the spatial average over $\Omega$ of a scalar field $F$. The field-based expression Eq. (29) of the effective impedance does not require the Bloch mode $\left(\boldsymbol{E}^{(2)}, \boldsymbol{H}^{(2)}\right)$ to be normalized in any particular way since the value of the ratio of the field averages does not change when $\left(\boldsymbol{E}^{(2)}, \boldsymbol{H}^{(2)}\right)$ is multiplied by a factor $C \neq 0$. But, although the reflection coefficient is still given by $R=\left(Z_{\text {rel }}-1\right) /\left(Z_{\text {rel }}+1\right)$, the precise expression for the transmission coefficient depends on how the Bloch modes of the media (1) and (2) are chosen.

For normal incidence, we have $Z^{(1)}=1$ and the relation Eq. (29) simplifies to

$$
Z_{\mathrm{eff}}^{(2)}=\left(J^{(0,0)}\right)^{2}=\frac{\left\langle E_{y}^{(2)}\right\rangle^{2}}{\left\langle\left(\boldsymbol{E}^{(2)} \times \boldsymbol{H}^{(2)}\right) \cdot \boldsymbol{e}_{z}\right\rangle},
$$

which has some similarity with the effective impedance definition proposed in [13], i.e., $Z_{\text {eff }}^{(2)}=\left|\left\langle E_{y}^{(2)}\right\rangle^{2}\right| /\left\langle\left(\boldsymbol{E}^{(2)} \times \boldsymbol{H}^{(2) *}\right)\right.$. $\left.\boldsymbol{e}_{z}\right\rangle$. Poor accuracy was reported [6] when the effective impedance [13] was used to compute the Fresnel reflectance of photonic crystals at non-normal incidence angle (see Figs. 21 and 23 of the paper [6]). This is probably due to the fact that the impedance formula Eq. (30) as well as the one in [13] are valid only for normal incidence or near normal incidence where $e^{i k_{x} x} \approx 1$.

The derivation of the effective impedance in [13] was based on the continuity of power across the interface between two media thus it is restricted to modes which can carry power away from the interface (propagating modes); in particular this effective impedance formalism would be invalid in a medium where the fundamental mode is evanescent (our impedance theory does not have such a limitation).

\section{Other definitions of effective impedance}

As pointed out in [15], the form of the approximate expressions Eqs. (20) and (21) for the reflection and transmission coefficients depends on the set of basis functions used for the projection of the rigorous modal expansions Eqs. (5) and (6) into the truncated system of linear equations Eqs. (16) and (17). The mathematical expression for the effective impedance and the corresponding average field formula depend on the selected projection. We have observed that many existing averaged fieldbased impedance formulas (see for instance Table 3 of the review article [6]) can be related to a scattering-based impedance formula with a specific choice of projection. 
For an example, if Eqs. (5) and (6) were projected respectively, onto $\boldsymbol{z} \times \boldsymbol{H}^{(2)+}$ and $\boldsymbol{z} \times \boldsymbol{E}^{(1)+}$ instead of $\boldsymbol{z} \times \boldsymbol{H}^{(1)+}$ and $z \times \boldsymbol{E}^{(2) \dagger}$, we are led to the following approximate expressions for $R$ and $T$

$$
\begin{aligned}
R & =\frac{1-L^{\left(k_{x}, k_{y}\right)} L^{\left(-k_{x},-k_{y}\right)}}{1+L^{\left(k_{x}, k_{y}\right)} L^{\left(-k_{x},-k_{y}\right)}}, \\
T & =\frac{2 L^{\left(k_{x}, k_{y}\right)}}{1+L^{\left(k_{x}, k_{y}\right)} L^{\left(-k_{x},-k_{y}\right)}},
\end{aligned}
$$

with

$$
\begin{aligned}
L^{\left(k_{x}, k_{y}\right)} & =\int_{\Omega} \boldsymbol{E}^{(1)} \cdot\left(\boldsymbol{z} \times \boldsymbol{H}^{(2) \dagger}\right) d A, \\
L^{\left(-k_{x},-k_{y}\right)} & =\int_{\Omega} \boldsymbol{E}^{(1) \dagger} \cdot\left(\boldsymbol{z} \times\left(-\boldsymbol{H}^{(2)}\right)\right) d A .
\end{aligned}
$$

This gives rise to the following definition of effective impedance

$$
Z_{\text {rel }}=\frac{1}{L^{\left(k_{x}, k_{y}\right)} L^{\left(-k_{x},-k_{y}\right)}} .
$$

With the mode-matching equations Eqs. (5) and (6) at full rank, the matrix form [5] of the definitions Eqs. (23) and (35) will give exactly the same relative effective impedance matrix. However the scalar relative effective impedance Eqs. (23) and (35) are not equal in general because they are based on truncated modematching equations.

By repeating the derivations leading to Eq. (29), we obtain the following averaged-field expression of $Z_{\mathrm{eff}}^{(2)}$ :

$$
Z_{\mathrm{eff}}^{(2)}=\frac{\left\langle\left(\boldsymbol{E}^{(2)}(x, y, 0) \times \boldsymbol{H}^{(2)}(-x, y, 0)\right) \cdot \boldsymbol{e}_{z}\right\rangle}{\left\langle H_{x}^{(2)}(x, y, 0) e^{-i k_{x} x}\right\rangle\left\langle H_{x}^{(2)}(-x, y, 0) e^{i k_{x} x}\right\rangle} .
$$

In particular, for normal incidence we have

$$
Z_{\mathrm{eff}}^{(2)}=\frac{1}{\left(L^{(0,0)}\right)^{2}}=\frac{\left\langle\left(\boldsymbol{E}^{(2)} \times \boldsymbol{H}^{(2)}\right) \cdot \boldsymbol{e}_{z}\right\rangle}{\left\langle H_{x}^{(2)}\right\rangle^{2}}
$$

which, again, has some similarity with the formula Eq. (23) of [14].

Up to now, we have chosen modes on both sides of the interface for the projection of the mode-matching equations Eqs. (5) and (6); but it is also possible to project only on the modes from one side of the interface. For instance, by projecting Eqs. (5) and (6) onto $\boldsymbol{z} \times \boldsymbol{H}^{(1) \dagger}$ and $\boldsymbol{z} \times \boldsymbol{E}^{(1) \dagger}$, respectively, we are led to the following results

$$
\begin{gathered}
R=\frac{J^{\left(k_{x}, k_{y}\right)}-L^{\left(-k_{x},-k_{y}\right)}}{J^{\left(k_{x}, k_{y}\right)}+L^{\left(-k_{x},-k_{y}\right)}}=\frac{J^{\left(k_{x}, k_{y}\right)} / L^{\left(-k_{x},-k_{y}\right)}-1}{J^{\left(k_{x}, k_{y}\right)} / L^{\left(-k_{x},-k_{y}\right)}+1}, \\
T=\frac{2}{J^{\left(k_{x}, k_{y}\right)}+L^{\left(-k_{x},-k_{y}\right)}}=\frac{2 / L^{\left(k_{x}, k_{y}\right)}}{J^{\left(k_{x}, k_{y}\right)} / L^{\left(-k_{x},-k_{y}\right)}+1}, \\
Z_{\mathrm{rel}}=\frac{J^{\left(k_{x}, k_{y}\right)}}{L^{\left(-k_{x},-k_{y}\right)}}, \\
Z_{\mathrm{eff}}^{(2)}=\frac{\left\langle E_{y}^{(2)} e^{-i k_{x} x}\right\rangle}{\left\langle H_{x}^{(2)} e^{-i k_{x} x}\right\rangle} .
\end{gathered}
$$

The field-averaging impedance formula Eq. (41) was proposed in [12] and it is already known that it can be obtained from the Fresnel-based impedance formalism [11, 15]. The computed effective impedance tends to be only weakly dependent on the choice of the projection (see for instance Fig. 15 of [6]) and in this work we will use the effective impedance concept based on Eq. (23).

\section{THE EFFECTIVE MEDIUM MODELLING OF METAMA- TERIALS}

Normal incidence from vacuum can be the natural setting for retrieving the material parameters of the medium (2), since it leads to simpler formulas than non-normal incidence. For example, when the medium (2) is a genuine homogeneous medium, its refractive index $n^{(2)}$ is directly equal to the modal effective index $k_{z}^{(2)} / k_{0}$ if the mode $\left(\boldsymbol{E}^{(2)}, \boldsymbol{H}^{(2)}\right)$ is propagating in normal direction, while for a wave propagation in medium (2) at an angle $\theta$, we have $k_{z}^{(2)} / k_{0}=n^{(2)} \cos \theta^{(2)}$, where $k_{z}^{(2)}$ is the propagation constant of the mode $\left(\boldsymbol{E}^{(2)}, \boldsymbol{H}^{(2)}\right)$; as shown in Appendix, the wave impedance for an arbitrary angle of incidence can also be obtained from the wave impedance under normal incidence by using simple analytic formulas. Thus in this work, we will focus on the properties of the effective impedance for a normal incidence from vacuum. Accordingly we suppose that the medium (1) and medium (2) correspond respectively to the vacuum and metamaterial.

For normal incidence, the effective index and effective impedance of vacuum are $n_{\text {eff }}^{(1)}=1$ and $Z_{\text {eff }}^{(1)}=1$. The value of $n_{\text {eff }}^{(2)}=k_{z}^{(2)} / k_{0}$ and $Z_{\text {eff }}^{(2)}=\left(J^{(0,0)}\right)^{2}$ can depend on the polarization of the incident plane wave; we have chosen the polarization directions which are aligned with the symmetry axes (which are the $x$ and $y$ directions, with the examples considered here). Once the effective parameters $n_{\text {eff }}^{(2)}$ and $Z_{\text {eff }}^{(2)}$ are retrieved, the effective dielectric permittivity $\varepsilon_{\text {eff }}^{(2)}$ and magnetic permeability $\mu_{\text {eff }}^{(2)}$ can be computed according to the relations $\varepsilon_{\mathrm{eff}}^{(2)}=n_{\mathrm{eff}}^{(2)} / Z_{\mathrm{eff}}^{(2)}$ and $\mu_{\mathrm{eff}}^{(2)}=n_{\mathrm{eff}}^{(2)} Z_{\mathrm{eff}}^{(2)}$.

When a plane wave is incident (angle of incidence $\theta^{(1)}$ ) from vacuum over the homogenized medium (2), by using the angular dependence relation Eq. (79), in Appendix, the Fresnel reflection and transmission coefficients for the TE-polarization (or $E_{y}$-polarization) can be written as

$$
\begin{aligned}
& R_{\mathrm{TE}}=\frac{Z_{\mathrm{eff}}^{(2)} \cos \theta^{(1)}-\cos \theta^{(2)}}{Z_{\mathrm{eff}}^{(2)} \cos \theta^{(1)}+\cos \theta^{(2)}}, \\
& T_{\mathrm{TE}}=\frac{2 Z_{\mathrm{eff}}^{(2)} \cos \theta^{(1)}}{Z_{\mathrm{eff}}^{(2)} \cos \theta^{(1)}+\cos \theta^{(2)}},
\end{aligned}
$$

while, from the relation Eq. (81) in Appendix, the Fresnel reflection and transmission coefficients for the TM-polarization (or $H_{y}$-polarization) takes the form

$$
\begin{aligned}
& R_{\mathrm{TM}}=\frac{Z_{\mathrm{eff}}^{(2)} \cos \theta^{(2)}-\cos \theta^{(1)}}{Z_{\mathrm{eff}}^{(2)} \cos \theta^{(2)}+\cos \theta^{(1)}}, \\
& T_{\mathrm{TM}}=\frac{2 Z_{\mathrm{eff}}^{(2)} \cos \theta^{(1)}}{Z_{\mathrm{eff}}^{(2)} \cos \theta^{(2)}+\cos \theta^{(1)}} .
\end{aligned}
$$


In Eqs. (42)-(45), $\theta^{(2)}$ is the angle of transmission and the value of $\cos \theta^{(2)}$ can be derived from the Snell's law $n_{\mathrm{eff}}^{(2)} \sin \theta^{(2)}=$ $\sin \theta^{(1)}$ as follows:

$$
\cos \theta^{(2)}=\left(1-\frac{\sin ^{2} \theta^{(1)}}{n_{\mathrm{eff}}^{(2)^{2}}}\right)^{1 / 2}
$$

The formulas Eqs. (42)-(45) are in agreement with the ones given in $[18,19]$. Note that, for the TE-polarization, the following electric field plane wave functions $\boldsymbol{E}^{(j)}=$ $(0,1,0) e^{i\left(k_{x} x \pm k_{z}^{(j)} z\right)}$, for $j=1,2$, are used in the derivation of Eqs. (42) and (43) while, for the TM-polarization, the magnetic field plane wave functions are $\boldsymbol{H}^{(j)}=(0,1,0) e^{i\left(k_{x} x \pm k_{z}^{(j)} z\right)}$, for $j=1,2$, are used in Eqs. (44) and (45). When the plane wave basis functions are normalized according to Eqs. (7) and (8), then the transmission coefficients must be chosen as specified by Eq. (21). The expressions for the reflection coefficients are independent from the chosen basis functions, since both the incident and reflected fields are written in the same plane wave basis.

For metamaterial structures where the material or geometry property varies periodically along the $z$-direction, the value of the propagation constant $k_{z}^{(2)}$ is not uniquely defined. In such cases the value of $k_{z}^{(2)}$ can be obtained from the Bloch factor $\kappa=e^{i k_{z}^{(2)}} d_{z}$ and $k_{z}^{(2)}$ may take any of the following values:

$$
k_{z}^{(2)}=\frac{\log \kappa}{i d_{z}}+\frac{2 p \pi}{d_{z}}, \quad \forall p \in \mathbb{Z} .
$$

The value of $\cos \theta^{(2)}$ in Eq. (46) depends on the choice of the integer $p$ used in the computation of the effective index $n_{\mathrm{eff}}^{(2)}=$ $k_{z}^{(2)} / k_{0}$. As a consequence, the values of the Fresnel reflection and transmission coefficients Eqs. (42)-(45) also depend on $p$. So it is important to choose the integer $p$ whose Fresnel coefficients Eqs. (42)-(45) best replicate the Fresnel scattering properties of the metamaterials. This way of choosing the logarithmic branch of $k_{z}^{(2)}$ is similar in principle to the approach proposed in [2]. In general, in the effective medium limit, the physically meaningful propagation constant $k_{z}^{(2)}$ has a small value and so coincides with the principal value of the logarithm in Eq. (47). In this work $p$ is therefore set to $p=0$ for all computed effective indices $n_{\mathrm{eff}}^{(2)}=k_{z}^{(2)} / k_{0}$.

The homogenized medium can also provide a simplified model for analyzing the surface modes at the interface between a dielectric and a metamaterial as well as the modes of metamaterials waveguides, e.g., a dielectric layer sandwiched between two metamaterials (see Fig. 2). For example, the surface modes at the interface between vacuum and the homogenized material, if they exist, correspond to a pole of the reflection coefficient such that $k_{x}>k_{0}$ (bound mode) and the dispersion equation can be derived by setting the denominator of $R_{\mathrm{TM}}$ or $R_{\mathrm{TE}}$ to zero: $Z_{\text {eff }}^{(2)} \cos \theta^{(2)}+\cos \theta^{(1)}=0$ or $Z_{\text {eff }}^{(2)} \cos \theta^{(1)}+\cos \theta^{(2)}=0$. The dispersion equation of a planar waveguide with a core sandwiched between two homogenized materials (see Fig. 2) can be written as $R^{2} e^{2 i \sqrt{k_{0}^{2}-k_{x}^{2}} h}=1$, where $k_{x}$ is the propagation constant of the waveguide mode and $h$ is the thickness of the waveguide core.
In the next sections, we apply our effective medium method to two examples. The first example [3] can be treated analytically while the second example [20] relies on Bloch modes which are computed numerically. We then compare the response of the metamaterial and the homogenized material under a wide range of incidence angles. The conditions under which a good agreement exists define the domain of validity for using the homogenized material as a simplified model for analyzing the existence of surface modes or waveguide modes and their dispersion relations.

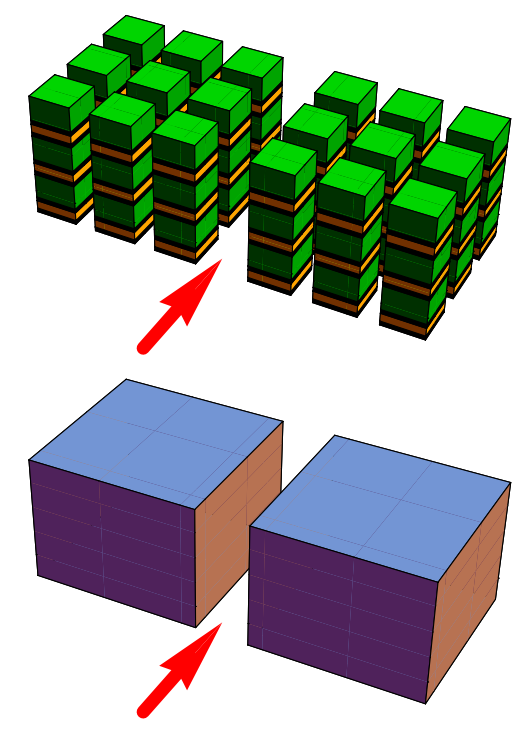

Fig. 2. Illustration of a planar waveguide with metamaterial cladding and a planar waveguide with homogenized material cladding.

\section{EXAMPLE OF A SURFACE WITH PERFECTLY CON- DUCTING HOLES}

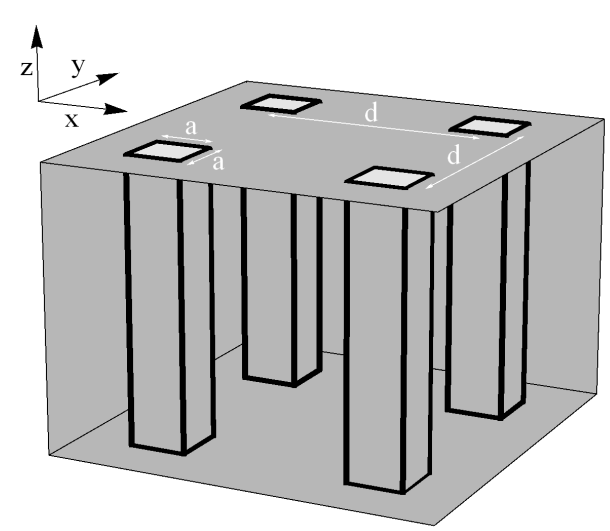

Fig. 3. A two-dimensional square array of holes perforated on a semi-infinite perfect conductor. The lattice constant is $d$ and the holes have a square cross-section $a \times a$.

As a test of validation, we apply our effective impedance technique to an example which has been previously analyzed in [3]. It is a two-dimensional square array of holes perforated on a semi-infinite perfect conductor (see Fig. 3). The cylinder 
holes have a square cross-section $[0, a] \times[0, a]$ and the waveguide modes inside these holes have a simple analytic expression. We assume that a plane wave is incident over the structure. In the long wavelength limit, i.e., $\lambda \gg d$, the contribution from the specular order will dominate the reflected field, while the fundamental eigenmode of the array is expected to dominate the transmitted field because it is the least decaying mode. Thus the modal expansion Eqs. (5)-(6) can be reduced to the contribution of the two dominant modes and the corresponding reflection and transmission coefficients can then be determined analytically [3].

The modes of the array take non-zero values only inside the holes. The transverse cross-section of the chosen unit cell is $(x, y) \in[0, d] \times[0, d]$ and the unit cell contains a hole with cross-section $[0, a] \times[0, a]$. The $E_{x}$-polarized fundamental mode $\left(\boldsymbol{E}^{(2)}, \boldsymbol{H}^{(2)}\right)$ can be written as

$$
\begin{aligned}
\boldsymbol{E}^{(2)} & =M^{(2)} e^{i k_{z}^{(2)} z} \sin \left(\frac{\pi y}{a}\right)\left[\begin{array}{l}
1 \\
0 \\
0
\end{array}\right], \\
\boldsymbol{H}^{(2)} & =\frac{-i}{k_{0} \mu} \nabla \times \boldsymbol{E}^{(2)} \\
& =\frac{M^{(2)} e^{i k_{z}^{(2)} z}}{k_{0} \mu}\left[\begin{array}{c}
k_{z}^{(2)} \sin \left(\frac{\pi y}{a}\right) \\
\frac{i \pi}{a} \cos \left(\frac{\pi y}{a}\right)
\end{array}\right],
\end{aligned}
$$

with $k_{z}^{(2)}=\sqrt{k_{0}^{2}-\pi^{2} / a^{2}}$. The corresponding adjoint mode can be obtained by applying the normalization Eq. (10): $\left(\boldsymbol{E}^{(2) \dagger}, \boldsymbol{H}^{(2) \dagger}\right)=\left(\boldsymbol{E}^{(2)},-\boldsymbol{H}^{(2)}\right)$. The factor $M^{(2)}$ in Eqs. (48) and (49) is chosen such that $\boldsymbol{E}^{(2)}$ and $\boldsymbol{H}^{(2) \dagger}$ satisfy the biorthogonality relation Eq. (7):

$$
M^{(2)}=\frac{1}{a} \sqrt{\frac{2 k_{0} \mu}{k_{z}^{(2)}}} .
$$

For normal incidence by an $E_{x}$-polarized plane wave, the mode $\left(\boldsymbol{E}^{(1)}, \boldsymbol{H}^{(1)}\right)$ of the incident medium can be taken as

$$
\begin{aligned}
& \boldsymbol{E}^{(1)}=M^{(1)} e^{i k_{0} z}\left[\begin{array}{l}
1 \\
0 \\
0
\end{array}\right], \\
& \boldsymbol{H}^{(1)}=\frac{-i}{k_{0} \mu} \nabla \times \boldsymbol{E}^{(1)}=M^{(1)} \frac{e^{i k_{0} z}}{\mu}\left[\begin{array}{l}
0 \\
1 \\
0
\end{array}\right] .
\end{aligned}
$$

In order to satisfy the biorthogonality relation Eq. (7), the factor $M^{(1)}$ in Eqs. (51) and (52) must be set to

$$
M^{(1)}=\frac{\sqrt{\mu}}{d} .
$$

The overlap integral at the interface $z=0$ between the fundamental modes of the media (1) and (2) is

$$
\begin{aligned}
J^{(0,0)} & =\int_{\Omega} \boldsymbol{E}^{(2)} \cdot\left(\boldsymbol{e}_{z} \times \boldsymbol{H}^{(1) \dagger}\right) d A, \\
& =\frac{M^{(1)} M^{(2)}}{\mu} \int_{0}^{a} \int_{0}^{a} \sin \left(\frac{\pi y}{a}\right) d x d y,
\end{aligned}
$$

i.e.,

$$
J^{(0,0)}=\frac{2 a}{\pi d} \sqrt{\frac{2 k_{0}}{k_{z}^{(2)}}} .
$$

So that the effective impedance of the lossless metamaterial at normal incidence is

$$
Z_{\text {eff }}^{(2)}(0,0)=\left(J^{(0,0)}\right)^{2}=\frac{8 a^{2} k_{0}}{\pi^{2} d^{2} k_{z}^{(2)}} .
$$

The expression Eq. (56) for the effective impedance at normal incidence is identical to the one given by Eq. (24) of [3]. The effective index is

$$
n_{\mathrm{eff}}^{(2)}=\frac{k_{z}^{(2)}}{k_{0}}=\frac{\sqrt{k_{0}^{2}-\pi^{2} / a^{2}}}{k_{0}},
$$

and for wavelengths $\lambda \gg a$, the effective index takes a large imaginary number, i.e., $n_{\mathrm{eff}}^{(2)} \approx i \pi /\left(k_{0} a\right)$.

We now study the case of non-normal incidence; we consider an incident TM-polarized (or $H_{y}$-polarization) plane wave with wave vector $k=\left(k_{x}, 0, k_{z}^{(1)}\right)$; the case of TE-polarization (or $E_{y}$-polarization) can also be treated in a similar manner. The plane wave basis function can be taken as

$$
\begin{aligned}
& \boldsymbol{E}^{(1)}=M^{(1)} e^{i\left(k_{x} x+k_{z}^{(1)} z\right)}\left[\begin{array}{c}
k_{z}^{(1)} \\
0 \\
-k_{x}
\end{array}\right], \\
& \boldsymbol{H}^{(1)}=\frac{-i}{k_{0} \mu} \nabla \times \boldsymbol{E}^{(1)}=M^{(1)} \frac{e^{i\left(k_{x} x+k_{z}^{(1)} z\right)}}{\mu}\left[\begin{array}{c}
0 \\
k_{0} \\
0
\end{array}\right],
\end{aligned}
$$

where we have used the relation $k_{z}^{(1)^{2}}+k_{x}^{2}=k_{0}^{2}$. The corresponding adjoint modes are obtained by applying Eqs. (11) and (12),

$$
\begin{aligned}
& \boldsymbol{E}^{(1) \dagger}=M^{(1)} e^{i\left(-k_{x} x+k_{z}^{(1)} z\right)}\left[\begin{array}{c}
k_{z}^{(1)} \\
0 \\
k_{x}
\end{array}\right], \\
& \boldsymbol{H}^{(1) \dagger}=M^{(1)} \frac{e^{i\left(-k_{x} x+k_{z}^{(1)} z\right)}}{\mu}\left[\begin{array}{c}
0 \\
-k_{0} \\
0
\end{array}\right],
\end{aligned}
$$

The biorthogonality property holds at the interface $z=0$ when the normalization factor $M^{(1)}$ is set to the value

$$
M^{(1)}=\frac{1}{d} \sqrt{\frac{\mu}{k_{0} k_{z}^{(1)}}} .
$$

The value of the overlap integral $J^{\left(k_{x}, 0\right)}$ is

$$
\begin{aligned}
J^{\left(k_{x}, 0\right)} & =\int_{\Omega} \boldsymbol{E}^{(2)} \cdot\left(\boldsymbol{e}_{z} \times \boldsymbol{H}^{(1) \dagger}\right) d A \\
& =\frac{M^{(1)} M^{(2)}}{\mu} \int_{0}^{a} k_{0} e^{-i k_{x} x} d x \int_{0}^{a} \sin \left(\frac{\pi y}{a}\right) d y \\
& =\frac{M^{(1)} M^{(2)} k_{0} e^{-i k_{x} a / 2}}{\mu} \\
& \times \int_{-a / 2}^{a / 2} e^{-i k_{x} x} d x \int_{0}^{a} \sin \left(\frac{\pi y}{a}\right) d y
\end{aligned}
$$


i.e.,

$$
J^{\left(k_{x}, 0\right)}=\frac{2 a k_{0} e^{-i k_{x} a / 2}}{\pi d} \sqrt{\frac{2}{k_{z}^{(1)} k_{z}^{(2)}}} \frac{\sin \left(k_{x} a / 2\right)}{k_{x} a / 2} .
$$

We note that the complex number $e^{-i k_{x} a / 2}$ in Eq. (66), which comes from the integration of $e^{-i k_{x} x}$ over the interval $[0, a]$, was omitted in Eq. (21) of [3].

For non-normal impedance, the effective wave impedance of vacuum is

$$
Z_{\text {eff }}^{(1)}\left(k_{x}, 0\right)=\frac{E_{x}}{H_{y}}=\frac{k_{z}^{(1)}}{k_{0}}=\cos \theta^{(1)},
$$

and so the effective wave impedance of the metamaterial is

$$
Z_{\text {eff }}^{(2)}\left(k_{x}, 0\right)=Z_{\text {rel }}\left(k_{x}, 0\right) Z_{\text {eff }}^{(1)}\left(k_{x}, 0\right)
$$

where the relative effective impedance $Z_{\text {rel }}\left(k_{x}, 0\right)$ is defined by Eq. (23):

$Z_{\text {rel }}\left(k_{x}, 0\right)=J^{\left(k_{x}, 0\right)} J^{\left(-k_{x}, 0\right)}=\frac{8 a^{2} k_{0}}{\pi^{2} d^{2} k_{z}^{(2)}} \frac{k_{0}}{k_{z}^{(1)}}\left(\frac{\sin \left(k_{x} a / 2\right)}{k_{x} a / 2}\right)^{2}$.

So that we have

$$
Z_{\mathrm{eff}}^{(2)}\left(k_{x}, 0\right)=Z_{\mathrm{eff}}^{(2)}(0,0)\left(\frac{\sin \left(k_{x} a / 2\right)}{k_{x} a / 2}\right)^{2},
$$

with $Z_{\text {eff }}^{(2)}(0,0)$ being the effective impedance at normal incidence Eq. (56). By comparison, the impedance for the $H_{y^{-}}$ polarization given by the homogenized material with effective impedance Eq. (56) and effective index Eq. (57) can be derived from the reflection coefficient Eq. (44) as:

$$
Z_{\text {eff }}^{(2)}\left(k_{x}, 0\right)=Z_{\text {eff }}^{(2)}(0,0) \cos \theta^{(2)},
$$

where the cosine of the angle of transmission $\theta^{(2)}$ is defined by Eq. (46) as follows:

$$
\begin{aligned}
\cos \theta^{(2)} & =\left(1-\frac{\sin ^{2} \theta^{(1)}}{n_{\mathrm{eff}}^{(2)^{2}}}\right)^{1 / 2}=\left(1-\frac{\left(k_{x} / k_{0}\right)^{2}}{\left(k_{z}^{(2)} / k_{0}\right)^{2}}\right)^{1 / 2} \\
& =\left(1+\frac{k_{x}^{2}}{\pi^{2} / a^{2}-k_{0}^{2}}\right)^{1 / 2} .
\end{aligned}
$$

In the long wavelength limit, the effective impedances Eq. (70) and Eq. (71) are equivalent since we have

$$
\left(1+\frac{k_{x}^{2}}{\pi^{2} / a^{2}-k_{0}^{2}}\right)^{1 / 2} \approx 1 \text { and }\left(\frac{\sin \left(k_{x} a / 2\right)}{k_{x} a / 2}\right)^{2} \approx 1
$$

and the homogenized material can be used to simulate the properties of the metamaterials. While the propagation constant $k_{z}^{(2)}$ of the mode Eqs. (48) and (49) does not depend on the transverse momentum $k_{x}$, the value of $k_{z}^{(2)}$ given by the homogenized material, i.e., $k_{z}^{(2)}=\left(n_{\mathrm{eff}}^{2} k_{0}^{2}-k_{x}^{2}\right)^{1 / 2}=$ $\left(k_{0}^{2}-\pi^{2} / a^{2}-k_{0}^{2} \sin ^{2} \theta^{(1)}\right)^{1 / 2}$, depends on $k_{x}=k_{0} \sin \theta^{(1)}$, although such dependence is negligible for long wavelengths where we have $k_{z}^{(2)} \approx i \pi / a$.
Surface modes at the interface between vacuum and the homogenized material, if they exist, correspond to the zeros of the denominator of $R_{\mathrm{TM}}$ (i.e., $Z_{\text {eff }}^{(2)} \cos \theta^{(2)}+\cos \theta^{(1)}=0$ ) or of $R_{\mathrm{TE}}$ (i.e., $Z_{\text {eff }}^{(2)} \cos \theta^{(1)}+\cos \theta^{(2)}=0$ ). Conditions for the existence of surface modes can be established by analyzing the signs of the effective dielectric permittivity $\varepsilon_{x, \mathrm{eff}}^{(2)}=\varepsilon_{y \text {,eff }}^{(2)}=n_{\mathrm{eff}}^{(2)} / Z_{\mathrm{eff}}^{(2)}$ and the magnetic permeability $\mu_{x, \text { eff }}^{(2)}=\mu_{y, \text { eff }}^{(2)}=n_{\text {eff }}^{(2)} Z_{\text {eff }}^{(2)}$; we have $\varepsilon_{x, \text { eff }}^{(2)}=\varepsilon_{y \text {,eff }}^{(2)}$ and $\mu_{x \text {,eff }}^{(2)}=\mu_{y, \text { eff }}^{(2)}$ since the $x$ and $y$ directions are symmetrically equivalent. If $\varepsilon_{\text {eff }}^{(1)}=1$ and $\varepsilon_{x, \text { eff }}^{(2)}$ are of opposite signs, then TM-polarized surface modes can exist while TE-polarized surface modes can exist when $\mu_{\text {eff }}^{(1)}=1$ and $\mu_{x, \text { eff }}^{(2)}$ have opposite signs. Here both effective parameters $Z_{\text {eff }}^{(2)}$ and $n_{\text {eff }}$ are imaginary complex numbers, with respectively negative and positive imaginary parts, which implies that $\varepsilon_{x, \text { eff }}^{(2)}=$ $\varepsilon_{y, \text { eff }}^{(2)}=n_{\text {eff }}^{(2)} / Z_{\text {eff }}^{(2)}$ is a negative number and the magnetic permeability $\mu_{x, \text { eff }}^{(2)}=\mu_{y, \text { eff }}^{(2)}=n_{\text {eff }}^{(2)} Z_{\text {eff }}^{(2)}$ is a positive number. It follows that surface modes with TM-polarization (or $H_{y}$-polarization or electric plasmon) can exist and the corresponding dispersion equation is $Z_{\text {eff }}^{(2)} \cos \theta^{(2)}+\cos \theta^{(1)}=0$. In the long wavelength limit, Eq. (72) shows that we have $\cos \theta^{(2)} \approx 1$, so that the dispersion equation for the electric surface plasmon becomes $\left(1-k_{x}^{2} / k_{0}^{2}\right)^{1 / 2}+Z_{\text {eff }}^{(2)}=0$, which is the same as the dispersion equation Eq. (29) in [3] (when the dielectric constant $\varepsilon_{\mathrm{h}}$ is equal to one) and an example of a dispersion curve can also be seen in Fig. 4 of [3].

The results about lossless metamaterials in this section show a complete agreement between our theory of effective impedance and the theory presented in [3]. Our definition of effective impedance has a general scope and can be applied also to lossy metamaterials and in the next section we will consider such a case.

\section{EXAMPLE OF A LOSSY METAMATERIAL}

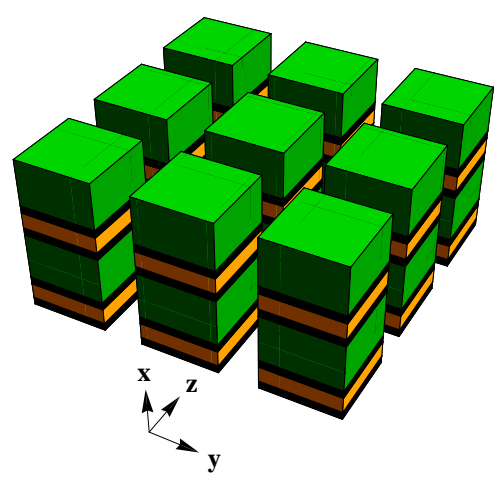

Fig. 4. The metamaterials [20] designed for operation in the frequency range $0.2 \mathrm{THz}$ to $0.4 \mathrm{THz}$.

As a model of a lossy metamaterial, we consider the example of a metamaterial structure consisting of an array of layered metal-dielectric-metal pillars, as illustrated in Fig. 4. Each pillar is a stack, oriented along the $x$ direction, of magnetic resonators separated by a low index dielectric material $\varepsilon_{2}=2.6$. A magnetic resonator consists of high index dielectric material 
$\varepsilon_{1}=90.0$ sandwiched between two parallel metallic plates (dielectric constant $\varepsilon_{\mathrm{m}}$ ). The metal is silver and the dielectric constant is obtained using the Drude model

$$
\varepsilon_{\mathrm{m}}(\omega)=1-\frac{\omega_{P}^{2}}{\omega(\omega+i \gamma)},
$$

with plasma frequency $\omega_{P}=9 \mathrm{eV}$ and scattering frequency $\gamma=$ $0.054 \mathrm{eV}$.

The lattice constants in the $x, y$ and $z$ directions are $d_{x}=$ $12.1 \mu \mathrm{m}, d_{y}=d_{z}=25 \mu \mathrm{m}$. The metamaterial system is designed for applications in the Terahertz regime [20]. Over the operating frequency band, the wavelength $\lambda$ is much longer than the lattice constant of the metamaterials and an effective medium method can be applicable. For instance, we have $8.56<\lambda / d_{y}<120$ over the frequency range $[0.1 \mathrm{THz}, 1.4 \mathrm{THz}]$.

\section{A. Effective parameters and scattering}

Figure 5 shows the computed effective index $n_{\mathrm{eff}}^{(2)}$ and effective impedance $Z_{\text {eff }}^{(2)}$, for normal incidence by an $\left(E_{x}, H_{y}\right)$-polarized plane wave; the corresponding effective dielectric permittivity $\varepsilon_{x, \text { eff }}^{(2)}=n_{\text {eff }}^{(2)} / Z_{\text {eff }}^{(2)}$ and effective magnetic permeability $\mu_{y, \text { eff }}=$ $\mu_{z \text {,eff }}^{(2)}=n_{\text {eff }}^{(2)} Z_{\text {eff }}^{(2)}$ are plotted in Fig. 6 (since the metamaterial structure has the same symmetry along the $y$ and $z$ directions, we have $\mu_{z \text {,eff }}^{(2)}=\mu_{y \text {,eff }}^{(2)}$. Similarly, Fig. 7 shows the computed effective index $n_{\text {eff }}^{(2)}$ and effective impedance $Z_{\text {eff }}^{(2)}$, for normal incidence by an $\left(E_{y}, H_{x}\right)$-polarized plane wave while the corresponding effective dielectric permittivity $\varepsilon_{y \text {,eff }}^{(2)}=\varepsilon_{z \text {,eff }}^{(2)}$ and effective magnetic permeability $\mu_{x \text {,eff }}^{(2)}$ are displayed in Fig 8.

The Fresnel reflectance for the TE-polarizations (or $E_{x^{-}}$ polarization) and TM-polarization (or $H_{x}$-polarization) are shown in Fig. 9, for the incidence angles $\theta_{0}=0^{\circ}, \theta_{0}=40^{\circ}$, $\theta_{0}=80^{\circ}$ and $\theta_{0}=85^{\circ}$. Note that we have adopted the axis convention used in [20]; in particular the components of the wave vector of the specular order are: $k_{x}=0, k_{y}=k_{0} \sin \theta_{0}$ and $k_{z}^{(1)}=k_{0} \cos \theta_{0}$. The continuous curves in Fig. 9 represent the reflectance of the metamaterial, computed using a highly accurate numerical method [9]. The dashed curves in Fig. 9 indicate the reflectance of the homogenized material; the reflectance is calculated with the formulas Eqs. (42) and (44). The results in Fig. 9 show that the homogenized material reproduces the reflection properties of the metamaterials over a wide range of angles of incidence; this suggests that the homogenized material waveguide can be used to simulate the dispersion curves of the guided modes of a metamaterial waveguide.

\section{B. Surface waves and waveguide modes}

Figure 6 indicates that the effective dielectric permittivity $\varepsilon_{x, \text { eff }}^{(2)}=n_{\text {eff }}^{(2)} / Z_{\text {eff }}^{(2)}$ and the effective magnetic permeability $\mu_{y, \text { eff }}^{(2)}=\mu_{z \text {,eff }}^{(2)}=n_{\text {eff }}^{(2)} Z_{\text {eff }}^{(2)}$ have respectively a positive and a negative real part for frequencies $v \in[0.75 \mathrm{THz}, 1.15 \mathrm{THz}]$ (because the metamaterial structure has the same symmetry along the $y$ and $z$ directions, we have $\mu_{z \text {,eff }}^{(2)}=\mu_{y \text {,eff }}^{(2)}$. It follows that the vacuum-homogenized material interface can support surface modes with TE-polarization (or $E_{x}$-polarization
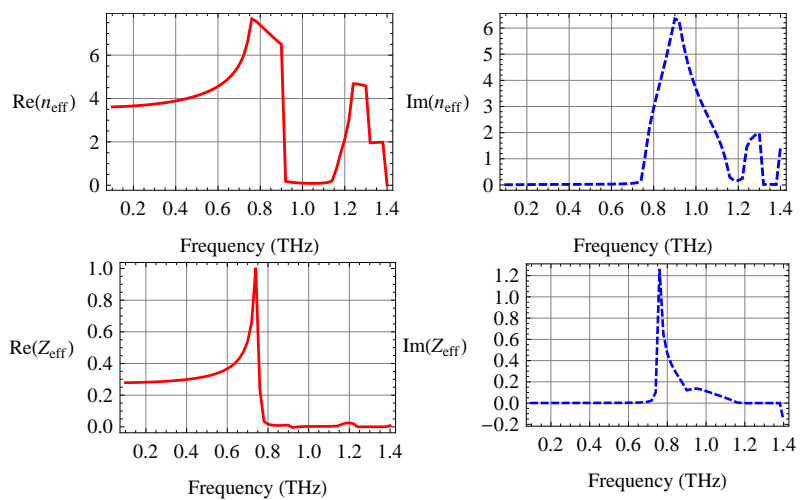

Fig. 5. The computed effective parameters $n_{\text {eff }}^{(2)}$ and $Z_{\text {eff }}^{(2)}$ of the metamaterials shown in Fig. 4. The scattering data for normal incidence, by an $E_{x}$-polarized plane wave, are used to obtain $n_{\text {eff }}^{(2)}$ and $Z_{\text {eff }}^{(2)}$.
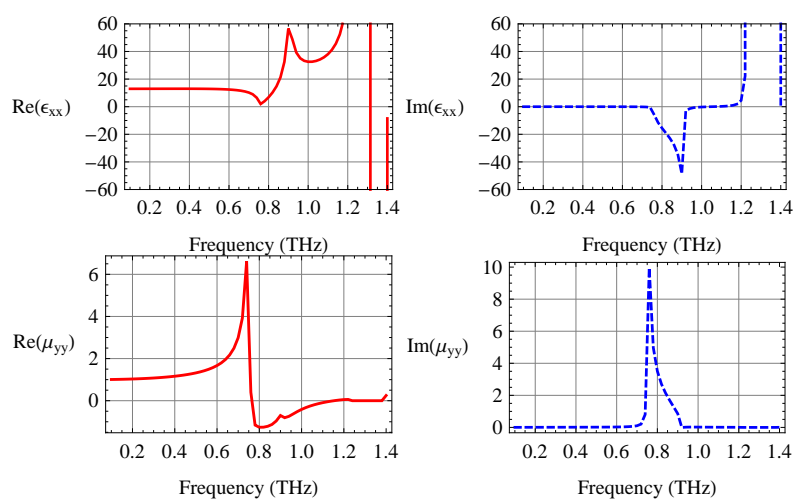

Fig. 6. The real and imaginary parts of the effective dielectric constant $\varepsilon_{x, \text { eff }}^{(2)}$ and effective magnetic permeability $\mu_{y, \text { eff }}^{(2)}$; they are obtained from normal incidence by an $E_{x}$-polarized plane wave.
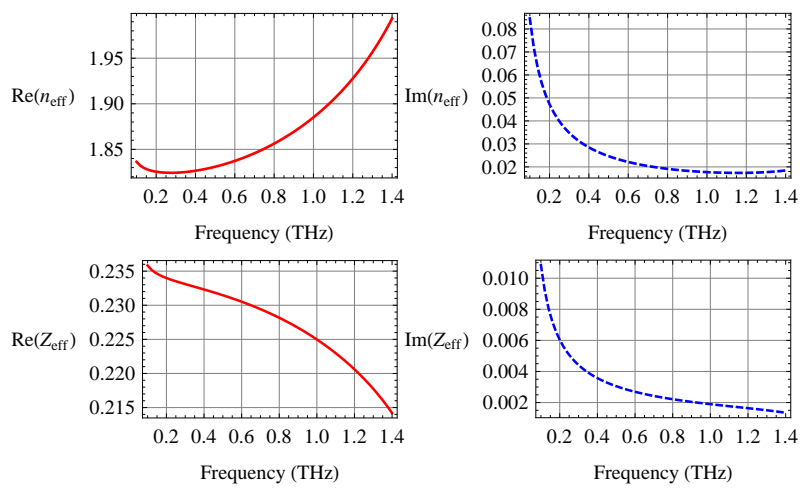

Fig. 7. The computed effective parameters $n_{\text {eff }}^{(2)}$ and $Z_{\text {eff }}^{(2)}$ of the metamaterials shown in Fig. 4, for normal incidence by an $E_{y^{-}}$ polarized plane wave. 


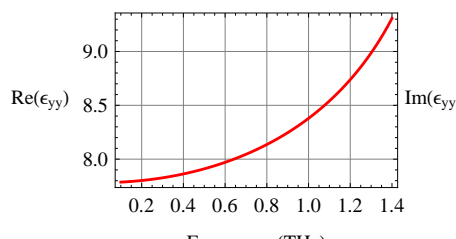

Frequency (THz)

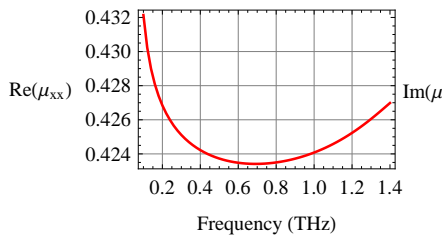

Frequency $(\mathrm{THz})$

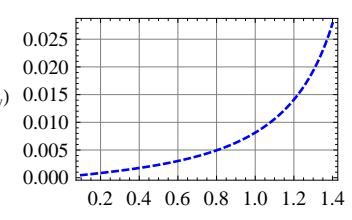

Frequency $(\mathrm{THz})$

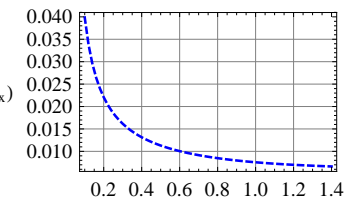

Frequency $(\mathrm{THz})$
Fig. 8. The real and imaginary parts of the effective dielectric constant $\varepsilon_{y, \text { eff }}^{(2)}$ and effective magnetic permeability $\mu_{x, \text { eff }}^{(2)}$. They are obtained from normal incidence by an $E_{y}$-polarized plane wave.
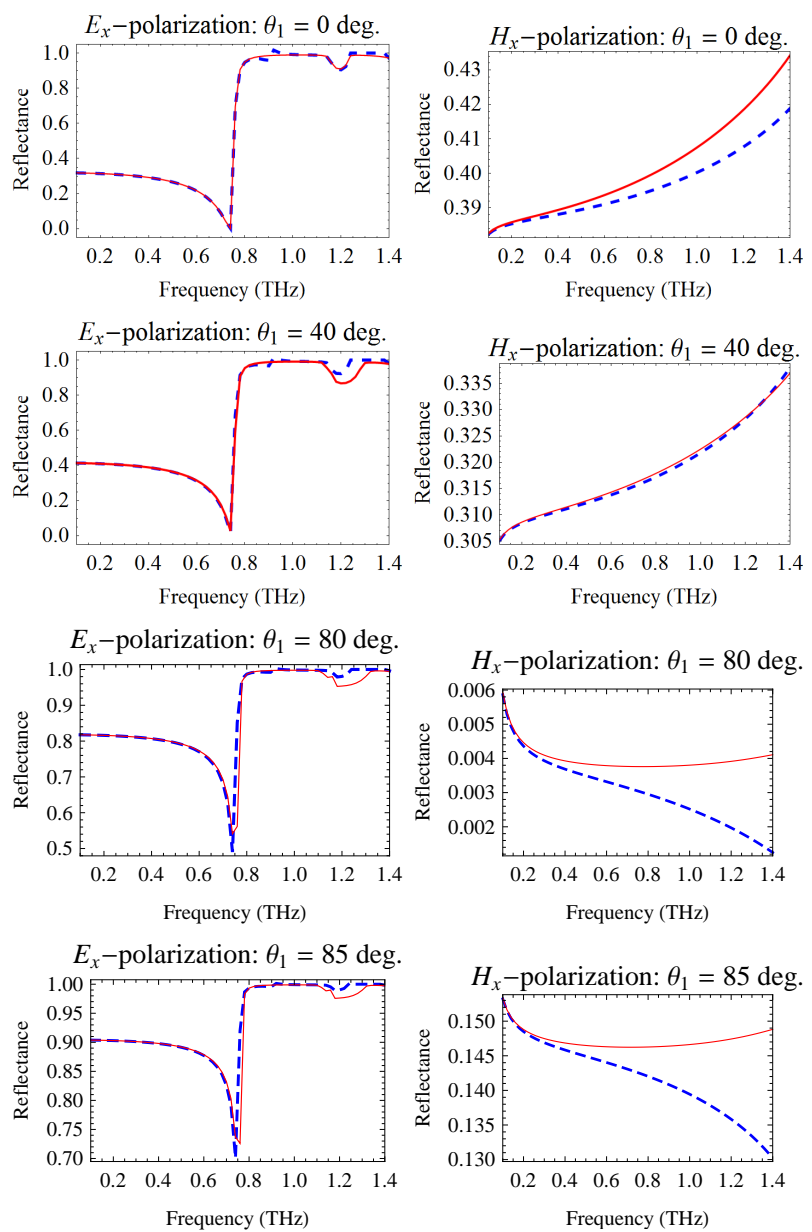

Fig. 9. Fresnel reflectance curves for the $E_{x}$ and $E_{y}$ polarized plane waves. The continuous curves correspond to the reflectance of the metamaterials. The dashed curves indicate the reflectance of the homogenized material. or magnetic surface plasmon) and the dispersion equation is $Z_{\text {eff }}^{(2)} \cos \theta^{(1)}+\cos \theta^{(2)}=0$.

The existence of a negative magnetic permeability (with the associated magnetic surface plasmons) was also shown in [20]. The presence of magnetic surface plasmons implies that a metamaterial waveguide can guide $E_{x}$-polarized waves. The field confinement near the waveguide core does not rely on total internal reflection; instead confinement is due to the existence of surface modes, so that a metamaterial waveguide with a subwavelength core of diameter $h$ can still confine waves. As an example, we consider a planar metamaterial waveguide with a vacuum core of diameter $h=38 \mu \mathrm{m}$; this is the same diameter value as in [20]. Here we consider a planar waveguide which reduces to a one-dimensional problem for the waveguide with a homogenized medium cladding, while for the metamaterial waveguide we have used a periodic boundary condition along the $x$-direction and a supercell consisting of 6 unit-cells on each side of the core, oriented along the $y$-direction.

(a)

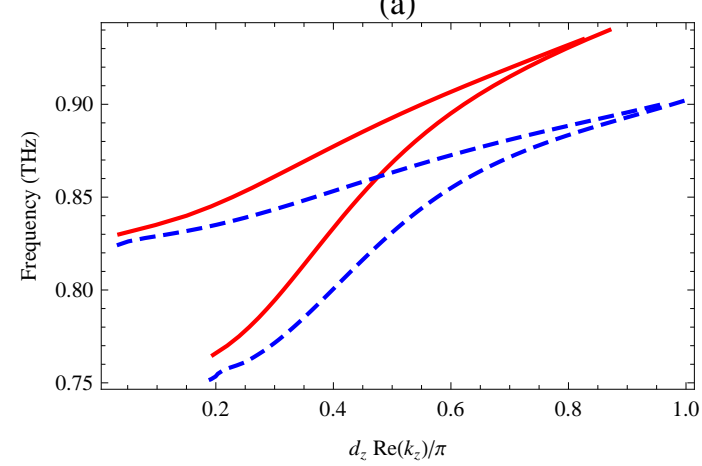

(b)

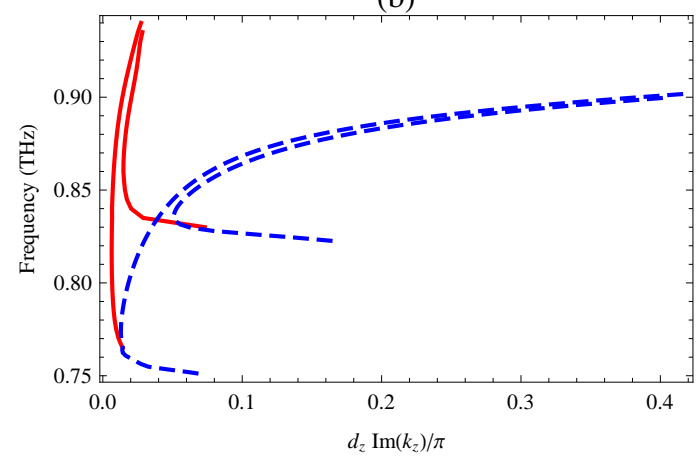

Fig. 10. The continuous and dashed curves are respectively the dispersion curves of the planar metamaterial waveguide and the planar homogenized material waveguide. (a) Dispersion of the frequency against the real part of $d_{z} k_{z} / \pi$. (b) Dispersion of the frequency against the imaginary part of $d_{z} k_{z} / \pi$.

In order to analyze the complex dispersion curves, we plot the frequency against the real part of $d_{z} k_{z} / \pi$ (normalized phase factor) in Fig. 10 (a), and against the imaginary part of $d_{z} k_{z} / \pi$ (normalized attenuation factor) in Fig. 10 (b). In Figs. 10 (a) and (b), the continuous curves and dashed curves indicate respectively the dispersion curves of the planar metamaterial waveguide and the homogenized material waveguide. The dispersion curves of the homogenized material waveguide is obtained by solving the transcendental equa- 
tions $R_{\mathrm{TE}}^{2} e^{2 i \sqrt{k_{0}^{2}-k_{y}^{2}} h}=1$ numerically; this equation can be split into an equation for even-symmetry modes (lower dashed curve in Fig. 10 (a)): $R_{\mathrm{TE}} e^{i \sqrt{k_{0}^{2}-k_{y}^{2}} h}=1$ and an equation for odd-symmetry modes (upper dashed curve in Fig. 10 (a)): $R_{\mathrm{TE}} e^{i \sqrt{k_{0}^{2}-k_{y}^{2}} h}=-1$. The dispersion equation of the metamaterial waveguide is solved over a supercell using the finite element method described in [9]; the unit cell consists of a single layer of the metamaterial element (i.e., no stack of layers in the $x$-direction) and the left and right cladding contains 6 unit cells each; by comparison we only need one unit cell to compute the Bloch modes which are used to compute the effective parameters $n_{\text {eff }}^{(2)}$ and $Z_{\text {eff }}^{(2)}$. Regarding the dispersion curves of the frequency against the real part of $k_{z}$ in Fig. 10 (a), the homogenized material waveguide replicates the main features of the metamaterial waveguide (continuous curve), with a relative discrepancy $\left(v_{\mathrm{H}}\left(\operatorname{Re}\left(k_{z}\right)\right)-v_{\mathrm{M}}\left(\operatorname{Re}\left(k_{z}\right)\right)\right) / v_{\mathrm{M}}\left(\operatorname{Re}\left(k_{z}\right)\right)$ less than $6 \%$, where $v=v_{\mathrm{H}}\left(\operatorname{Re}\left(k_{z}\right)\right)$ and $v=v_{\mathrm{M}}\left(\operatorname{Re}\left(k_{z}\right)\right)$ denote respectively the dispersion relation of the homogenized material waveguide and the metamaterial waveguide. The calculation of the dispersion curves of the homogenized material waveguide takes only a small fraction of the computation time and memory required for the metamaterial waveguide (once the effective parameters are determined). However for the dispersion curves of the frequency against the imaginary part of $k_{z}$ in Fig. 10 (b), the effective medium results and the finite element results are close near the lower cut-off but deviate significantly as the frequency increases. This shows that the effective medium technique is less accurate for the computation of attenuation loss of metamaterial waveguides.

\section{Brewster angle for metamaterials}

With the $H_{x}$-polarization, the metamaterials behave like a dielectric medium with a positive effective dielectric constant $\varepsilon_{y, \text { eff }}=\varepsilon_{z \text {,eff }}$ and a positive effective magnetic permeability $\mu_{x, \text { eff }}$; metamaterials can also exhibit some interesting properties, such as the existence of a Brewster angle. For instance, we can observe in Fig. 9 that, for the incidence angle $\theta_{0}=80^{\circ}$ the reflectance for the $H_{x}$-polarization has very small values, i.e., lower than 0.006. A Brewster angle, if it exists, is an angle of incidence $\theta_{0}=\theta_{\mathrm{Br}}$ such that either of the reflection coefficient Eqs. (42) or (44) is zero. By solving the corresponding equations, the Brewster angle for the $H_{y}$ and $E_{y}$-polarizations can be written as [19]:

$$
\begin{aligned}
& \theta_{\mathrm{Br}, \mathrm{TM}}=\arcsin \left(\sqrt{\frac{1-Z_{\mathrm{eff}}^{(2)^{2}}}{1-Z_{\mathrm{eff}}^{(2)^{2}} / n_{\mathrm{eff}}^{(2)^{2}}}}\right), \\
& \theta_{\mathrm{Br}, \mathrm{TE}}=\arcsin \left(\sqrt{\frac{1-Z_{\mathrm{eff}}^{(2)^{2}}}{1 / n_{\mathrm{eff}}^{(2)^{2}}-Z_{\mathrm{eff}}^{(2)^{2}}}}\right) .
\end{aligned}
$$

For incidence by an $H_{x}$-polarized plane wave, if $n_{\text {eff }}$ and $Z_{\text {eff }}^{(2)}$ are positive numbers such that $n_{\text {eff }}^{(2)}>1$ and $Z_{\text {eff }}^{(2)}<1$, then we can verify that the angle $\theta_{\mathrm{Br}, \mathrm{TM}}$ in Eq. (75) exists as a real number (since $\left|1-Z_{\text {eff }}^{(2)^{2}}\right| \leq\left|1-Z_{\text {eff }}^{(2)^{2}} / n_{\text {eff }}^{(2)^{2}}\right|$ ). As it appears in Fig. 5, for the $H_{y}$-polarization, $n_{\text {eff }}^{(2)}$ and $Z_{\text {eff }}^{(2)}$ have a small imaginary part while their real parts satisfy the conditions $\operatorname{Re}\left(n_{\text {eff }}^{(2)}\right)>1$ and $\operatorname{Re}\left(Z_{\text {eff }}^{(2)}\right) \leq 1 / \operatorname{Re}\left(n_{\text {eff }}^{(2)}\right)<1$, accord- ingly we can expect the homogenized materials to have a physically relevant Brewster angle $\theta_{\mathrm{Br}, \mathrm{TM}}$ with a small imaginary part.

The real and imaginary parts of the Brewster angle $\theta_{\mathrm{Br}, \mathrm{TM}}$ given by Eq. (75) are plotted in Fig.11; the real part of $\theta_{\mathrm{Br}, \mathrm{TM}}$ is close to $80^{\circ}$, i.e., $\operatorname{Re}\left(\theta_{\mathrm{Br}, \mathrm{TM}}\right) \in\left[78.4891^{\circ}, 79.2582^{\circ}\right]$ and, as expected, the imaginary part is relatively small, i.e., $\operatorname{Im}\left(\theta_{\mathrm{Br}, \mathrm{TM}}\right) \in$ $\left[-0.7623^{\circ},-0.101524^{\circ}\right]$; this is consistent with the fact that the reflectance has small value when the angle of incidence is $80^{\circ}$. Note that the Brewster angle $\theta_{\mathrm{Br} \text {, TE }}$ for the $E_{y}$-polarization has a large imaginary over most of the frequency range considered in Fig. 5 and it is not plotted here.
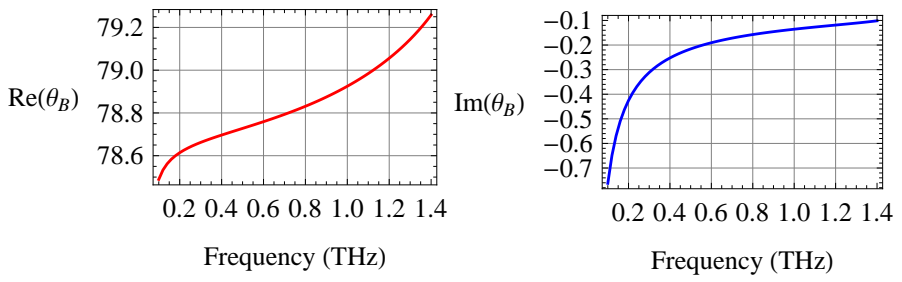

Fig. 11. Real and imaginary parts of the Brewster angles for the $H_{x}$-polarization.

\section{CONCLUSION}

We have developed a technique for retrieving the effective impedance of metamaterials from the Fresnel scattering coefficient at the interface between two semi-infinite media and have implemented a simple semi-analytic modelling technique based on the effective impedance. Fresnel scattering-based approaches for retrieving the effective impedance can also be seen as field averaging techniques. In particular, the mathematical justifications for the effective impedance definitions based on Fresnel reflection coefficients can also be extended to some effective impedance definitions based on the field averaging techniques (by using an appropriate choice of the projection operators). The effective medium approach is a computationally efficient numerical method (fast computation and reduced memory usage requirement) and so it can be an attractive modelling tool when it produces results with an acceptable accuracy. We have shown that it can accurately model the field scattering at the interface between dielectric media and metamaterials within the effective medium limit, e.g., when the ratio between wavelength and the lattice constant of the metamaterials is sufficiently high. For the metamaterial waveguides considered here, the effective medium approach can replicate the waveguide dispersion curves (frequency versus phase factor) with a relative discrepancy less than $6 \%$ when compared to an accurate numerical simulation of the metamaterial waveguides using a finite element technique. For the dispersion curves of frequency versus attenuation factor, the effective medium approach produces accurate results only near the waveguide cut-off.

\section{APPENDIX: ANGULAR DEPENDENCE OF THE WAVE IMPEDANCE OF HOMOGENEOUS MEDIA}

The wave impedance of a homogeneous medium depends on the direction of propagation of the corresponding plane wave. In this section we will show that once the wave impedance for normal incidence is known, the wave impedance for any 
angle of propagation can be obtained using some simple formulas. We consider a homogeneous medium (2) with a dielectric permittivity $\varepsilon_{\mathrm{eff}}^{(2)}$, a magnetic permeability $\mu_{\mathrm{eff}}^{(2)}$ and a refractive index $n^{(2)}=\sqrt{\varepsilon_{\mathrm{eff}}^{(2)} \mu_{\mathrm{eff}}^{(2)}}$. When a plane wave is incident with angle of incidence $\theta^{(1)}$ from vacuum onto the homogenized medium (2), the angle of transmission $\theta^{(2)}$ can be derived from the conservation of tangential momentum: $k_{x}=n^{(2)} k_{0} \sin \theta^{(2)}=k_{0} \sin \theta^{(1)}$ and this leads to Snell's law $n^{(2)} \sin \theta^{(2)}=\sin \theta^{(1)}$.

For incidence by an $E_{y}$-polarized plane wave, the mode $\left(\boldsymbol{E}^{(2)}, \boldsymbol{H}^{(2)}\right)$ takes the form $\boldsymbol{E}^{(2)}=\left(0, E_{y}^{(2)}, 0\right) e^{i\left(k_{x} x+k_{z}^{(2)} z\right)}$ and $\boldsymbol{H}^{(2)}=\left(H_{x}^{(2)}, 0, H_{z}^{(2)}\right) e^{i\left(k_{x} x+k_{z}^{(2)} z\right)}$, where

$$
k_{z}^{(2)}=\left(\left(n^{(2)} k_{0}\right)^{2}-k_{x}^{2}\right)^{1 / 2}=k_{0} n^{(2)} \cos \theta^{(2)} .
$$

From the definition of wave impedance and the Maxwell's equation Eq. (13), we have for $Z_{\text {eff }}\left(k_{x}, k_{y}\right)$

$$
Z_{\text {eff }}^{(2)}\left(k_{x}, 0\right)=\frac{E_{y}^{(2)}}{-H_{x}^{(2)}}=\frac{k_{0} \mu^{(2)}}{k_{z}^{(2)}}=\frac{\mu^{(2)}}{n^{(2)} \cos \theta^{(2)}},
$$

so that we have the angular dependence

$$
Z_{\text {eff }}^{(2)}\left(k_{x}, 0\right)=\frac{Z_{\text {eff }}^{(2)}(0,0)}{\cos \theta^{(2)}} .
$$

Note that the wave impedance Eq. (78) can also be derived from Eq. (29). We can verify that the wave impedance, for incidence by $H_{y}$-polarized plane wave, is

$$
Z_{\text {eff }}^{(2)}\left(k_{x}, 0\right)=\frac{E_{x}^{(2)}}{H_{y}^{(2)}}=\frac{k_{z}^{(2)}}{k_{0} \epsilon^{(2)}}=\frac{n^{(2)} \cos \theta^{(2)}}{\epsilon^{(2)}},
$$

which leads to the angular dependence

$$
Z_{\text {eff }}^{(2)}\left(k_{x}, 0\right)=Z_{\text {eff }}^{(2)}(0,0) \cos \theta^{(2)} .
$$

\section{ACKNOWLEDGMENTS}

This research was conducted by the Australian Research Council Centre of Excellence for Ultrahigh Bandwidth Devices for Optical Systems (project number CE110001018). This research was undertaken with the assistance of resources from the National Computational Infrastructure (NCI), which is supported by the Australian Government.

\section{REFERENCES}

1. D. R. Smith, S. Schultz, P. Markoš, and C. M. Soukoulis, "Determination of effective permittivity and permeability of metamaterials from reflection and transmission coefficients," Phys. Rev. B 65, 195104 (2002).

2. X. Chen, T. M. Grzegorczyk, B.-I. Wu, J. Pacheco, and J. A. Kong, "Robust method to retrieve the constitutive effective parameters of metamaterials," Phys. Rev. E 70, 016608 (2004).

3. F. J. Garcia-Vidal, L. Martín-Moreno, and J. B. Pendry, "Surfaces with holes in them: new plasmonic metamaterials," J. Opt. A: Pure Appl. Opt. 7, S97 (2005).

4. J. Yang, C. Sauvan, T. Paul, C. Rockstuhl, F. Lederer, and P. Lalanne, "Retrieving the effective parameters of metamaterials from the single interface scattering problem,” Appl. Phys. Lett. 97, 061102 (2010).
5. K. B. Dossou, L. C. Botten, and C. G. Poulton, "Semi-analytic impedance modeling of three-dimensional photonic and metamaterial structures," J. Opt. Soc. Am. A 30, 2034-2047 (2013).

6. F. J. Lawrence, C. M. de Sterke, L. C. Botten, R. C. McPhedran, and K. B. Dossou, "Modeling photonic crystal interfaces and stacks: impedance-based approaches," Adv. Opt. Photon. 5, 385-455 (2013).

7. F. J. Lawrence, L. C. Botten, K. B. Dossou, and C. Martijn de Sterke, "Antireflection coatings for two-dimensional photonic crystals using a rigorous impedance definition," Appl. Phys. Lett. 93, 121114 (2008).

8. R. Mittra, Y.-L. Hou, and V. Jamnejad, "Analysis of open dielectric waveguides using mode-matching technique and variational methods," IEEE Trans. Microw. Theory Tech. 28, 36-43 (1980).

9. K. B. Dossou, L. C. Botten, A. A. Asatryan, B. C. P. Sturmberg, M. A. Byrne, C. G. Poulton, R. C. McPhedran, and C. M. de Sterke, "Modal formulation for diffraction by absorbing photonic crystal slabs," J. Opt. Soc. Am. A 29, 817-831 (2012).

10. D. R. Smith and J. B. Pendry, "Homogenization of metamaterials by field averaging," J. Opt. Soc. Am. B 23, 391-403 (2006).

11. W. Śmigaj and B. Gralak, "Validity of the effective-medium approximation of photonic crystals," Phys. Rev. B 77, 235445 (2008).

12. Z. Lu and D. W. Prather, "Calculation of effective permittivity, permeability, and surface impedance of negative-refraction photonic crystals," Opt. Express 15, 8340-8345 (2007).

13. B. Momeni, A. A. Eftekhar, and A. Adibi, "Effective impedance model for analysis of reflection at the interfaces of photonic crystals," Opt. Lett. 32, 778-780 (2007).

14. B. Momeni, M. Badieirostami, and A. Adibi, "Accurate and efficient techniques for the analysis of reflection at the interfaces of threedimensional photonic crystals," J. Opt. Soc. Am. B 24, 2957-2963 (2007).

15. T. Paul, C. Menzel, W. Śmigaj, C. Rockstuhl, P. Lalanne, and F. Lederer, "Reflection and transmission of light at periodic layered metamaterial films," Phys. Rev. B 84, 115142 (2011).

16. K. B. Dossou and L. C. Botten, "A combined three-dimensional finite element and scattering matrix method for the analysis of plane wave diffraction by bi-periodic, multilayered structures," J. Comput. Phys. 231, 6969-6989 (2012).

17. P. Lorrain, D. R. Corson, and F. Lorrain, Electromagnetic fields and waves (Freeman, New York, 1988), 3rd ed.

18. J. D. Jackson, Classical electrodynamics (John Wiley \& Sons Inc., New York, 1999), 3rd ed.

19. A. Sihvola, "Geometric visualization of the Brewster angle from dielectric-magnetic interface," Optik 117, 317-320 (2006).

20. A. Ishikawa, S. Zhang, D. A. Genov, G. Bartal, and X. Zhang, "Deep subwavelength Terahertz waveguides using gap magnetic plasmon," Phys. Rev. Lett. 102, 043904 (2009). 